\title{
POWER SUPPLY CONTROL AND MONITORING FOR THE SNS RING AND TRANSPORT SYSTEM*
}

\author{
R. Lambiase ${ }^{\dagger}$, B. Oerter, S. Peng, J. Smith, BNL, Upton, NY 11973, USA
}

\begin{abstract}
There are approximately 300 magnet power supplies in the SNS accumulator ring and transport lines. Control and monitoring of the these converters will be primarily accomplished with a new Power Supply Interface and Controller (PSI/PSC) system developed for the SNS project. This PSI/PSC system provides all analog and digital commands and status readbacks in one fiber isolated module. With a maximum rate of $10 \mathrm{KHz}$, the PSI/PSC must be supplimented with higher speed systems for the wide bandwidth pulsed injection supplies, and the even wider bandwidth extraction kickers. This paper describes the implementation of this PSI/PSC system, which was developed through an industry/laboratory collaboration, and the supplimentary equipment used to support the wider bandwidth pulsed supplies.
\end{abstract}

\section{OVERVIEW}

The SNS Project is a collaboration of six partner laboratories. Portions of the Project are being designed and constructed by each of the partner labs, and will then be integrated at the SNS site at Oak Ridge National Lab.

BNL is providing equipment for the accumulator ring and transport lines. This paper will describe how the control system was designed to simplify the procurement, testing, installation, operation and maintenance of the power supplies of that system.

The primary method of controlling the SNS power supplies is the Power Supply Interface (PSI) / Power Supply Controller (PSC) system. The PSI / PSC system concepts were developed about one year ago, and described in an earlier paper [1]. Over this past year, much progress has been made:

- The PSI / PSC has gone from concept to first article production, in a cooperative effort with Apogee Labs, Inc of N. Wales, PA.

- Extension of the PSI / PSC system to wide bandwidth and fast pulsed power supplies was developed.

- Software was developed to test the PSI / PSC performance, test power supply performance, and operate the power supplies for SNS.

\footnotetext{
*Work performed under the auspices of the U.S. Department of Energy. †lambiase@bnl.gov
}

\section{PSI / PSC}

The PSI / PSC system is all that is required to control the DC power supplies. Each PSC (controller) can control up to up to six PSIs (interfaces). It's major features are:

- One 16-bit analog output, four 16-bit analog inputs, fifteen digital commands, and sixteen digital readbacks.

- Timed readings and setpoints - by either hardware or software triggers.

- Burst mode - to determine ripple and other higher frequency components at up to a $10 \mathrm{KHz}$ rate.

- Circular buffer - to store about 5,000 sets of historical data.

- Isolation by fiber - to avoid isolation amplifiers.

- Data access by VME or serial port - to allow power supply testing with or without a complete control system.

\subsection{Arrangement for DC Power Supplies}

Of the approximately 300 power supplies in the accumulator ring and transfer lines, all but 22 are controlled with the basic configurations shown in Figure 1.

The front end computer and other modules, like the event decoder, reside in the I/O Crate (IOC). In normal operation, communication to each PSC is over the VME bus. Each PSC controls up to six PSIs, and each PSI controls a single power supply.

Each power supplies has electrical isolation from all others by using fiber optic cables between the PSI and the PSC. Each power supply has it's own circular buffer residing in the PSC that stores approximately 5,000 sets of data (readbacks and status bits). At the normal sampling rate of $60 \mathrm{~Hz}$, this provides over a minute of history, if stopped by a power supply fault.

If higher frequency information is desired, the PSC can be put into the burst mode where the buffer can be filled at a $10 \mathrm{KHz}$ rate. In a typical application, this would give good time resolution to look at the $720 \mathrm{~Hz}$ fundamental frequency of the ripple of a twelve pulse phase controlled power supply.

The connection between the PSI and the power supply is made with two cables: one for the analog signals, and a second for the digital signals. This simplifies installation. 


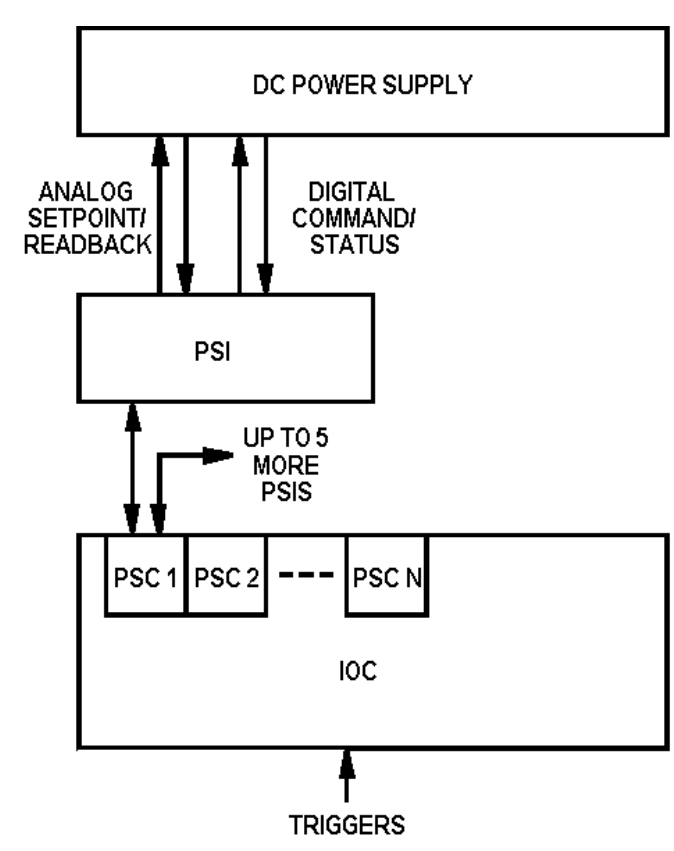

Figure 1: DC Power Supply Control Arrangement.

\subsection{Arrangement for Injection Bump Supplies}

The eight injection bump power supplies operate in two quadrants. The largest of these are rated at $\pm 800 \mathrm{~V}$ at 1400 Amps. All injection bump power supplies have a bandwidth of $2 \mathrm{KHz}$, requiring the slightly different control arrangement shown in Figure 2.

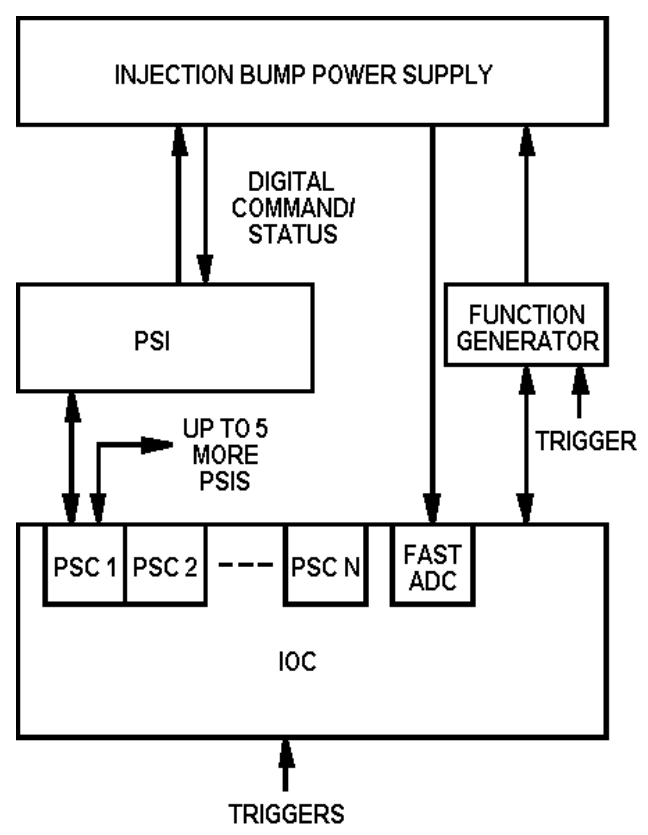

Figure 2: Injection Bump Supply Control Arrangement.
Here, the PSI provides only the digital control functions: status and command. The analog setpoint is supplied by a $100 \mathrm{KHz}$ function generator, and the four readbacks (setpoint current, measured current, error current, and voltage) are read by a $100 \mathrm{KHz} \mathrm{A} / \mathrm{D}$ converter residing in the $\mathrm{IOC}$.

\subsection{Arrangement for Extraction Kicker Supplies}

The third type of control arrangement is used for the fourteen extraction kickers. This is shown in Figure 3.

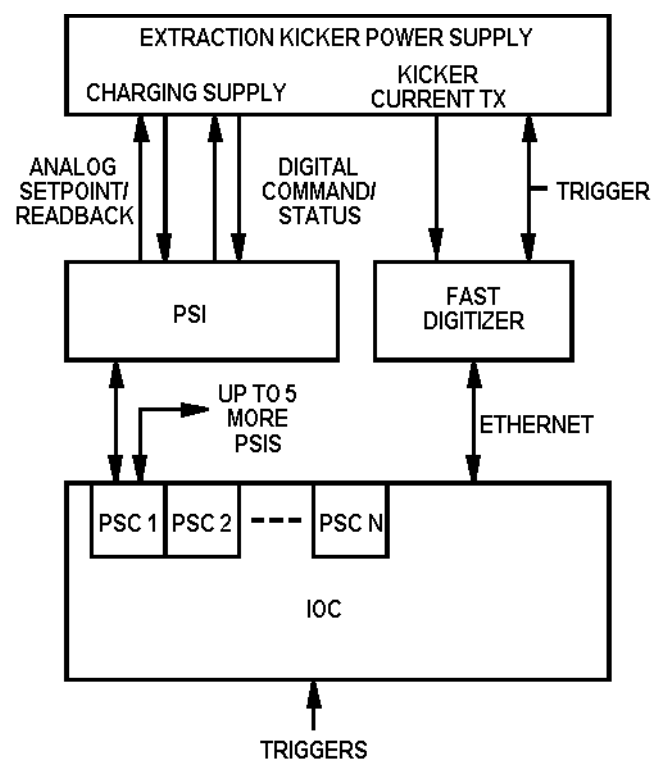

Figure 3: Extraction Kicker Control Arrangement.

From a control viewpoint, there are two parts to the extraction kicker power supplies. The charging power supply is low bandwidth, and is controlled by the PSI as the DC power supply. But, monitoring the current pulse, which has a $200 \mathrm{nSec}$ rise time requires a very fast digitizer. For this purpose, a 200-300 MHz, 8-bit digitizer is required. The specific device has not yet been selected - a digital scope may be used.

The connection between the fast digitizer and the IOC will be accomplished by either ethernet or GPIB.

\section{TEST CONFIGURATIONS}

A very important feature of the PSI / PSC based power supply control system is it's portability and stand-alone capability due to a serial port on the PSC, which provides an alternative to using VME based control.

\subsection{General Test Configuration}

The general test configuration is shown in Figure 4. 


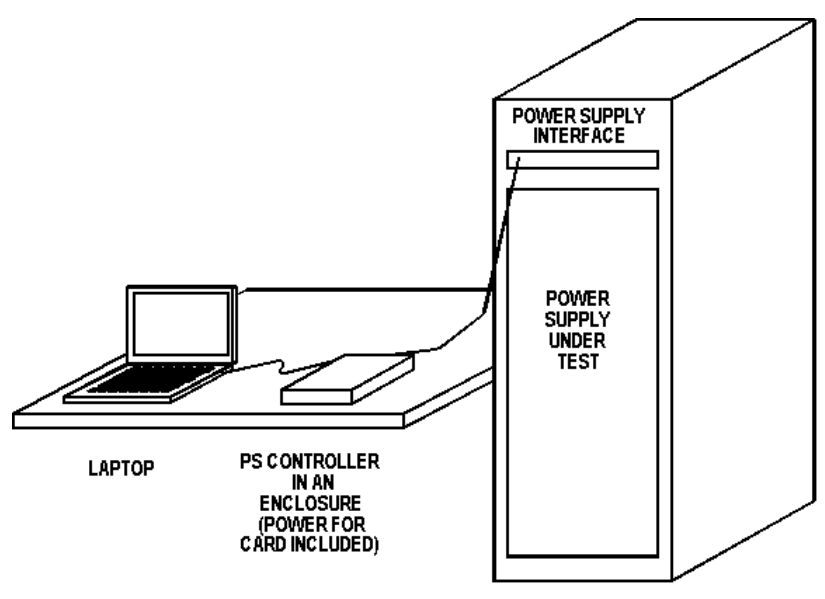

Figure 4: General Test Configuration.

For general testing purposes, an internally powered PSC is mounted in a chassis. Aside from this PSC box, the only other requirements to test a DC power supply is to have a PSI in that supply, and a laptop connected to the PSC box with a serial cable

\subsection{Testing During Power Supply Manufacture}

There are major advantages of this general test configuration during the manufacturing:

- The functionality of the controls interface is verified at the manufacturer. There are no compatibility problems to work out after the units have been shipped.

- This provides an automated system to the power supply manufacturer. This facilitates production testing and monitoring during burn-in.

- Test data is stored electronically, making archiving easier.

DC power supplies only need the PSI / PSC hardware. Injection bump supplies and extraction kickers will need the supporting equipment as well.

There are currently two types of SNS power supplies being constructed at outside vendors. The first article of the high power model of the injection bump power supply is to be tested during the last week of June, 2001. This test will need a function generator and fast readback capability as well as the PSI / PSC.

The second power supply now being developed at an outside vendor is the low field corrector. These units are rated at $\pm 20 \mathrm{~A}$ at $\pm 75 \mathrm{~V}$, and will be configured as six units to a standard rack. A single PSC and laptop will be used to control all six power supplies in the rack simultaneously.

\subsection{Incoming Test and On-Site Calibration}

The same advantages of the stand-alone test configuration for the manufacturers of the power supply, apply for the end users as well.

The laptop software is written using LabView (TM). This makes it easy to add other components used for test and calibration, including current, voltage, and temperature sensors.

\section{SUMMARY}

The SNS power supply controls were designed to have all the features required for most supplies in a single, stand-alone system. Other supplies, which had greater bandwidths are an extension of this system.

The control system simplifies not only installation, commissioning and operation of the machine, but also testing of the power supplies during manufacture and calibration.

The PSI and PSC components are currently in first article testing, and full production is scheduled for the end of June, 2001.

\section{REFERENCES}

[1] R. Lambiase, B. Oerter, J. Smith, "Power Supply Control for the Spallation Neutron Source," EPAC'00, Vienna, June 2000. 